\title{
A CONVENIENT SYNTHESIS OF PYRAZOLO[3,4-c]PYRAZOLES USING SOME NOVEL $\alpha$-CYANOKETENE DITHIOACETALS
}

Galal H. Elgemeie ${ }^{a *}$, Hosny A. Ali ${ }^{\mathrm{b}}$, Ahmed H. Elghandour ${ }^{\mathrm{b}}$ and Ahmed M. Hussein ${ }^{\mathrm{b}}$

a) Chemistry Department, Faculty of Science, Helwan University, Ain-Helwan, Cairo, Egypt

b) Chemistry Department, Faculty of Science, Cairo University (Bani Suef Branch), Bani Suef, Egypt

Abstract: A novel synthesis of pyrazolo[3,4-c]pyrazoles utilizing some novel $\alpha$-cyanoketene dithioacetals has been discussed.

Ketene dithioacetals bearing electron-withdrawing groups are versatile reagents for the synthesis of heterocycles and are extensively used'. In general, the ketene dithioacetals are obtained by the reaction of the corresponding active methylene compounds with carbon disulfide in the presence of a base, followed by the alkylation with alkylating agents such as methyl iodide. Among these compounds, ketene dithioacetals bearing the cyano or alkoxycarbonyl group at the $\alpha$-position are extremely interesting electrophilic reagents for the introduction of not only an ethenyl group or an acrylate moiety into amines, active methylene compounds, but also three or two carbon units into the ring of heterocyclic compounds ${ }^{2}$. During the course of our studies directed toward exploring the synthetic potential of ketene dithioacetals for synthesizing new classes of novel antimetabolites ${ }^{3}$, we have recently reported different successful approaches for synthesis of mercaptopurine and thioguanine analogues by the reaction of ketene dithioacetals with diazoles containing amino and active methylene functions ${ }^{4.5}$. In an extension of this work, we now report a synthesis of some novel ketene dithioacetals and their use for synthesis of functionalized pyrazolo[3,4-c]pyrazole derivatives. Thus, it has been found that reaction of 1cyanoacetyl-4-arylidenesemicarbazides 1 with carbon disulfide in the presence of sodium ethoxide gives the corresponding sodium dithiolate derivatives 2 in high yields. The latters on treatment with methyl iodide gives the novel ketene dithioacetals 3 . The structures of $\mathbf{3}$ have been established on the basis of their elemental analysis and spectral data. Thus, structure $\mathbf{3 b}$ is supported by its mass spectrum which showed a molecular formula $\mathrm{C}_{14} \mathrm{H}_{15} \mathrm{~N}_{3} \mathrm{OS}_{2}\left(\mathrm{M}^{+}=305\right)$ and ${ }^{1} \mathrm{H} \mathrm{NMR}$, which revealed two singlet bands at $\delta=2.32$ and $2.44 \mathrm{ppm}$. assignable to two methlysulfanyl groups and another singlet at $\delta=2.48$ assignable to one methyl group. The ylidenic proton is characterized by a singlet at $\delta=8.18 \mathrm{ppm}$. Another singlet appeared at $\delta=14.06 \mathrm{ppm}$. assignable to NH group. Reaction of compounds 3 with hydrazine derivatives 4 in a molar ratio $1: 2$ in refluxing ethanol containing catalytic amounts of piperidine or by fusion at $180^{\circ} \mathrm{C}$ gave the corresponding pyrazolo[3,4-c]pyrazoles 5 . The structures of 5 were established on the basis of their elemental analysis and spectral data (IR, 'H NMR, ${ }^{13} \mathrm{C}$ NMR and MS). The analytical data for 5e revealed a molecular formula $\mathrm{C}_{23} \mathrm{H}_{19} \mathrm{~N}_{7}\left(\mathrm{M}^{+}=393\right)$. The ${ }^{\prime} \mathrm{H} N M R$ spectrum revealed a broad singlet at $\delta=6.75 \mathrm{ppm}$. assignable to an amino group, a multiplet at $\delta=7.07$ $7.66 \mathrm{ppm}$. assigned to aromatic protons, a singlet at $\delta=7.87$ related to the $\mathrm{CH}$ group, and another singlet at $\delta=10.27 \mathrm{ppm}$. assignable to $\mathrm{NH}$ group. The ${ }^{13} \mathrm{C}$ NMR spectrum was characterized by a signals at $\delta=$ 111.93 and 118.63 corresponding to the C-1a and C-3a atoms. The signals at $\delta=125.49-128.95$ are assignable to the aromatic-carbons, while the signals appeared at $\delta=135.76,136.36$ and 145.22 are attributed to the ylidenic $\mathrm{CH}, \mathrm{C}-3$ and $\mathrm{C}-4$, respectively. The formation of 5 from the reaction of 3 with 4 is assumed to proceed via intermediacy of acyclic Michael adducts, which cyclized via addtion to the cyano group.

In summary, we have achieved a regiospecific synthesis of interesting pyrazolo[ $3,4-c]$-pyrazoles by the reaction of some novel ketene dithioacetals with hysrazines. The compounds obtained seem promising as high potential antimetabolite agents. 


\section{Experimental}

All melting points are uncorrected on a Gallenkamp melting point apparatus. The IR spectra were recorded ( $\mathrm{KBr}$ disk) on a Perkin Elmer 11650 FT-IR instrument. The ${ }^{1} \mathrm{H}$ NMR spectra were measured on a Varian $400 \mathrm{MHz}$ spectrometer for solutions in $\left(\mathrm{CD}_{3}\right)_{2} \mathrm{SO}$ using $\mathrm{Si}\left(\mathrm{CH}_{3}\right)_{4}$ as an internal standard. Mass spectra were recorded on a Varian MAT 112 spectrometer. Analytical data were obtained from the Microanalytical Data Center at Cairo University.

\section{Sodium dithiolate derivatives (2a-d)}

General procedure: A mixture of the 1-cyanoacetyl-4-arylidenesemicarbazide derivatives la- $d(0.01$ mole) and sodium ethoxide ( 0.02 mole) in absolute ethanol

$(30 \mathrm{ml})$ was gently heated for 30 minutes. The mixture was cooled, and then carbon disulphide $(0.01$ mole) was added gradually with stirring. The reaction mixture was heated for 15 minutes. The resulted sodium salt was precipetated by concentrating the solvent over water bath, then filtered off and recrystallized from the appropriate solvent.

2a: Yellow; m.p., > $300{ }^{\circ} \mathrm{C}$; from ethanol; yield, $61 \%$; $v_{\max } / \mathrm{cm}^{-1}(\mathrm{KBr}) 3422,3311(\mathrm{NH}), 1698(\mathrm{CO})$; found: C, 43.11; H, 2.42; N, $13.23 \%$; calcd. for $\mathrm{C}_{11} \mathrm{H}_{7} \mathrm{~N}_{3} \mathrm{OS}_{2} \mathrm{Na}_{2}$ (307); C, 42.99; H, 2.28; N, $13.68 \%$. 2b: Yellow; m.p., > $300{ }^{\circ} \mathrm{C}$; from ethanol; yield, $62 \%$; $v_{\max } / \mathrm{cm}^{-1}(\mathrm{KBr}) 3342,3245(\mathrm{NH}), 1696(\mathrm{CO})$; found: $\mathrm{C}, 45.00 ; \mathrm{H}, 2.51 ; \mathrm{N}, 12.82 \%$; calcd. for $\mathrm{C}_{12} \mathrm{H}_{9} \mathrm{~N}_{3} \mathrm{OS}_{2} \mathrm{Na}_{2}$ (321): C, 44.86; H, 2.80; N, $13.08 \%$. 2c: Yellow; m.p., > $300{ }^{\circ} \mathrm{C}$; from ethanol; yield, $68 \%$; $v_{\max } / \mathrm{cm}^{-1}(\mathrm{KBr}) 3411,3341$ (NH), 1700 (CO); found: $\mathrm{C}, 42.32 ; \mathrm{H}, 2.86 ; \mathrm{N}, 12.31 \%$; calcd. for $\mathrm{C}_{12} \mathrm{H}_{9} \mathrm{~N}_{3} \mathrm{O}_{2} \mathrm{~S}_{2} \mathrm{Na}_{2}$ (337); C, 42.73; H, 2.67; N, $12.46 \%$. 2d: Yellow; m.p., > $300{ }^{\circ} \mathrm{C}$; from ethanol; yield, $72 \%$; $v_{\max } / \mathrm{cm}^{-1}(\mathrm{KBr}) 3326,3224(\mathrm{NH}), 1689$ (CO); found: $\mathrm{C}, 38.26 ; \mathrm{H}, 1.70 ; \mathrm{N}, 12.41 \%$; calcd. for $\mathrm{C}_{11} \mathrm{H}_{6} \mathrm{~N}_{3} \mathrm{OS}_{2} \mathrm{ClNa}_{2}$ (341.5): C, 38.65; H, 1.75; N, 12.29 $\%$.

\section{Ketene-S,S-acetal derivatives (3a-d)}

General procedure: Methyl iodide ( 0.02 mole) was added to a solution of the

dithiolate sodium salts $2 \mathrm{a}-\mathbf{d}(0.01 \mathrm{~mol})$ in methanol $(30 \mathrm{ml})$ and the reaction mixture was stirred for 10 minutes. The formed solid product was collected by filtration and recrystallized from the appropriate solvent.

3a: Colorless; m.p., > $300{ }^{\circ} \mathrm{C}$; from ethanol; yield, $71 \%$; $v_{\max } / \mathrm{cm}^{-1}(\mathrm{KBr}) \quad 3455,3348(\mathrm{NH}) ; 2206(\mathrm{CN})$; 1712 (CO); $m / 2$ (291); found: C, 54.1; $\mathrm{H}, 4.2 ; \mathrm{N}, 14.1 \%$; calcd. for $\mathrm{C}_{13} \mathrm{H}_{13} \mathrm{~N}_{3} \mathrm{OS}_{2}$ (291): C, 53.61; $\mathrm{H}$, 4.46; N, $14.43 \%$. 3b: Orange; m.p., $>300{ }^{\circ} \mathrm{C}$; from ethanol; yield, $72 \%$; $v_{\max } / \mathrm{cm}^{-1}(\mathrm{KBr}) 3442-3173$ (NH), $2219(\mathrm{CN}), 1677(\mathrm{CO}) ;{ }^{1} \mathrm{H}$ NMR: $2.32\left(\mathrm{~s}, 3 \mathrm{H}, \mathrm{CH}_{3}\right) ; 2.44\left(\mathrm{~s}, 3 \mathrm{H}, \mathrm{SCH}_{3}\right) ; 2.48\left(\mathrm{~s}, 3 \mathrm{H}, \mathrm{SCH}_{3}\right) ; 7.20$ $7.61\left(\mathrm{~m}, 4 \mathrm{H}, \mathrm{C}_{6} \mathrm{H}_{4}\right) ; 8.18(\mathrm{~s}, 1 \mathrm{H}, \mathrm{CH}) ; 14.06(\mathrm{~s}, 1 \mathrm{H}, \mathrm{NH}) ;{ }^{13} \mathrm{C} \mathrm{NMR}: 18.22\left(\mathrm{CH}_{3}\right), 21.00\left(2 \mathrm{SCH}_{3}\right)$, $114.00(\mathrm{CN}), 120.70-129.50$ (aromatic-C), $132.50(\mathrm{C}-1), 139.66(\mathrm{CH}) ; 143.79$ (C-2); 162.89 (CO); found: C, 54.6; H, 5.1; N, $14.2 \%$; caled. for $\mathrm{C}_{14} \mathrm{H}_{13} \mathrm{~N}_{3} \mathrm{OS}$ (305): C, 55.08; H, 4.92; N, $13.77 \%$. 3c: Orange; m.p., $297^{\circ} \mathrm{C}$; from ethanol; yield, $69 \%$; $v_{\text {max }} / \mathrm{cm}^{-1}$ (KBr) 3481-3192 (NH), 2189 (CN), 1704 (CO); found: $\mathrm{C}, 52.6 ; \mathrm{H}, 4.9 ; \mathrm{N}, 12.9 \%$; calcd. for $\mathrm{C}_{14} \mathrm{H}_{15} \mathrm{~N}_{3} \mathrm{O}_{2} \mathrm{~S}_{2}$ (321): C, 52.33; H, 4.67; N, $13.08 \%$. 3d: Yellow; m.p., $285^{\circ} \mathrm{C}$; from ethanol; yield, $75 \%$; $v_{\max } / \mathrm{cm}^{-1}$ (KBr) 3348, 3229 (NH), 2221 (CN), 1695 (CO); found: $\mathrm{C}, 48.0 ; \mathrm{H}, 3.4 ; \mathrm{N}, 13.2 \%$; calcd. for $\mathrm{C}_{13} \mathrm{H}_{12} \mathrm{~N}_{3} \mathrm{OS} 2 \mathrm{Cl}$ (325.5): C, 47.93; H, 3.68; N, 12.90 $\%$.

\section{3-Substituted-4-amino-1 $H, 6 H$-pyrazolo[3,4-c]pyrazoles (5a-d)}

General procedure: A mixture of the ketene dithioacetals $3 \mathrm{a}-\mathrm{d}(0.01 \mathrm{~mole})$ and hydrazine hydrate $(0.02$ mole) was refluxed for $6 \mathrm{~h}$. in ethanol $(20 \mathrm{ml})$ containing a catalytic amount of piperidine. The solvent 
<smiles>[15CH]/C=N/NC(=O)CC#N</smiles>

1

$$
\begin{aligned}
& \mathrm{CS}_{2}-\mathrm{C}_{2} \mathrm{H}_{5} \overline{\mathrm{O}} \mathrm{Na}^{+} \\
& \mathrm{C}_{2} \mathrm{H}_{5} \mathrm{OH}-\mathrm{r} . \mathrm{t} .
\end{aligned}
$$<smiles>N#C/C=N/NC(=O)C(C#N)=C([As])[As]</smiles>

$\left.\right|^{2} \mathrm{CH}_{3} \mathrm{I}-\mathrm{C}_{2} \mathrm{H}_{5} \mathrm{OH}$<smiles>CC([SeH])=C(C#N)C(=O)N/N=C/[Bi]</smiles>

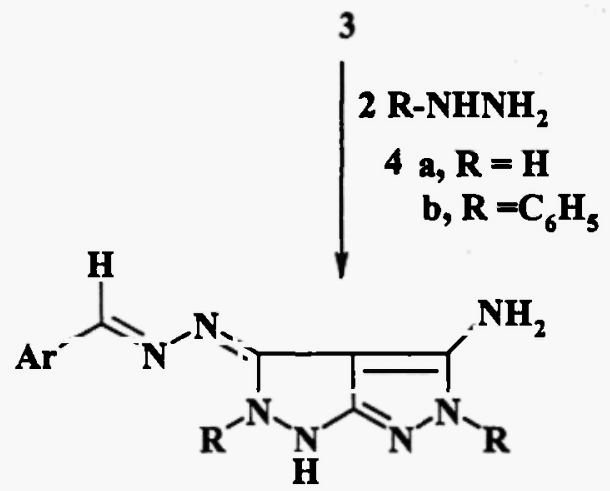

5

Chart ( 1)

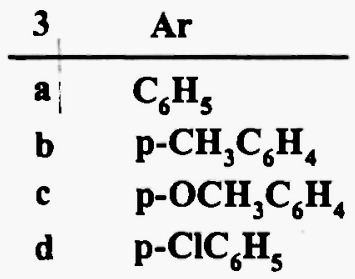

\begin{tabular}{llllll}
5 & $\mathrm{Ar}$ & $\mathrm{R}$ & 5 & $\mathrm{Ar}$ & $\mathrm{R}$ \\
\hline $\mathrm{a}$ & $\mathrm{C}_{6} \mathrm{H}_{5}$ & $\mathrm{H}$ & $\mathrm{e}$ & $\mathrm{C}_{6} \mathrm{H}_{5}$ & $\mathrm{C}_{6} \mathrm{H}_{5}$ \\
b & $\mathrm{p}-\mathrm{CH}_{3} \mathrm{C}_{6} \mathrm{H}_{4}$ & $\mathrm{H}$ & $\mathrm{f}$ & $\mathrm{p}-\mathrm{CH}_{3} \mathrm{C}_{6} \mathrm{H}_{4}$ & $\mathrm{C}_{6} \mathrm{H}_{5}$ \\
c & $\mathrm{p}-\mathrm{OCH}_{3} \mathrm{C}_{6} \mathrm{H}_{4}$ & $\mathrm{H}$ & $\mathrm{g}$ & $\mathrm{p}-\mathrm{OCH}_{3} \mathrm{C}_{6} \mathrm{H}_{4}$ & $\mathrm{C}_{6} \mathrm{H}_{5}$ \\
d & $\mathrm{p}-\mathrm{ClC}_{6} \mathrm{H}_{5}$ & $\mathrm{H}$ & $\mathrm{h}$ & $\mathrm{p}-\mathrm{ClC}_{6} \mathrm{H}_{5}$ & $\mathrm{C}_{6} \mathrm{H}_{5}$
\end{tabular}


was concentrated and the reaction mixture was neutralized with dilute $\mathrm{HCl}$. The product formed was collected by filtration and recrystallized from the appropriate solvent.

5a: Colorless; m.p., $187{ }^{\circ} \mathrm{C}$; from ethanol; yield, $64 \%$; $v_{\max } / \mathrm{cm}^{-1}(\mathrm{KBr}) 3415-3115\left(\mathrm{NH}, \mathrm{NH}_{2}\right) ; \mathrm{m} / \mathrm{z}$ (241); found: $\mathrm{C}, 54.1 ; \mathrm{H}, 4.8 ; \mathrm{N}, 39.3 \%$; calcd. for $\mathrm{C}_{11} \mathrm{H}_{11} \mathrm{~N}_{7}(241)$ : C, 54.77; H, 4.56; N, $40.66 \%$. 5b: Yellow; m.p., $212^{\circ} \mathrm{C}$; from ethanol-DMF; yield, $62 \%$; $v_{\max } / \mathrm{cm}^{-1}(\mathrm{KBr}) 3410-3165\left(\mathrm{NH}, \mathrm{NH}_{2}\right)$; found: $\mathrm{C}$, 56.2; H, 4.9; N, 39.0\%; calcd. for $\mathrm{C}_{12} \mathrm{H}_{13} \mathrm{~N}_{7}(255)$ : C, 56.47; H, 5.09; N, $38.43 \%$. 5c: Yellow; m.p., 234 ${ }^{\circ} \mathrm{C}$; from ethanol-DMF; yield, $68 \%$; $v_{\max } / \mathrm{cm}^{-1}(\mathrm{KBr}) 3398-3151\left(\mathrm{NH}, \mathrm{NH}_{2}\right)$; found: $\mathrm{C}, 53.4 ; \mathrm{H}, 4.9 ; \mathrm{N}$, $35.9 \%$; calcd. for $\mathrm{C}_{12} \mathrm{H}_{13} \mathrm{~N}_{7} \mathrm{O}$ (271): C, 53.14; H, 4.79; N, 36.16\%. 5d: Pale yellow; m.p., $211{ }^{\circ} \mathrm{C}$; from ethanol; yield, $75 \%$; $v_{\max } / \mathrm{cm}^{-1}(\mathrm{KBr}) 3460-3365(\mathrm{NH}) ; 3115\left(\mathrm{NH}_{2}\right) ;{ }^{1} \mathrm{H}$ NMR: 7.52-7.85 (b, 4H, $\left.\mathrm{C}_{6} \mathrm{H}_{4}\right)$; 7.98 (s, br, 2H, NH $)$ ); 8.75 (s, 1H, CH);10.42-10.61 (3s, br, 3H, 3NH); ${ }^{13} \mathrm{C}$ NMR: 113.25 (C-3a, C-1a); 128.26-130.05 (aromatic-C); $135.26(\mathrm{CH}) ; 138.74$ (C-3); 150.03 (C-4);-m/2 (275); found: C, 47.4; H, 3.2; $\mathrm{N}, 36.0 \%$; calcd. for $\mathrm{C}_{11} \mathrm{H}_{10} \mathrm{~N}_{7} \mathrm{Cl}$ (275.5): $\mathrm{C}, 47.91 ; \mathrm{H}, 3.63 ; \mathrm{N}, 35.57 \%$.

\section{3-Supstituted-4-amino-2,5-diphenyl-(1H)-pyrazole[3,4-c]pyrazoles (5e-h)}

General procedure: A mixture of ketene dithioacetals $3 \mathrm{a}-\mathrm{d}(0.01 \mathrm{~mole})$ and phenylhydrazine $(0.02$ mole) was heated at $160-170^{\circ} \mathrm{C}$ for 30 minutes on an oil bath. The reaction mixture was poured on an ice-water mixture $(100 \mathrm{ml})$ and neutralized with dilute $\mathrm{HCl}$. The formed solid product was collected by filtration and recrystallized from the appropriate solvent.

5e: Colorless, m.p., $162{ }^{\circ} \mathrm{C}$; from ethanol; yield, $65 \%$; $\nu_{\max } / \mathrm{cm}^{-1}(\mathrm{KBr}) 3425-3244\left(\mathrm{NH}, \mathrm{NH}_{2}\right) ;{ }^{1} \mathrm{H}$ NMR: $6.75\left(\mathrm{~s}, \mathrm{br}, 2 \mathrm{H}, \mathrm{NH}_{2}\right) ; 7.06-7.66\left(\mathrm{~m}, 15 \mathrm{H}, 3 \mathrm{C}_{6} \mathrm{H}_{5}\right) ; 7.87(\mathrm{~s}, 1 \mathrm{H}, \mathrm{CH}) ; 10.27(\mathrm{~s}, 1 \mathrm{H}, \mathrm{NH}) ;{ }^{13} \mathrm{C} \mathrm{NMR}$ : 111.93 (C-1a); 118.63 (C-3a); 125.49-128.95 (aromatic-C); 135.76 (CH); 136.36 (C-3); 145.22 (C-4); $\mathrm{m} / z$ (393); found: $\mathrm{C}, 71.0 ; \mathrm{H}, 4.9 ; \mathrm{N}, 24.3 \%$; calcd. for $\mathrm{C}_{23} \mathrm{H}_{19} \mathrm{~N}_{7}$ (393): C, 70.23; H, 4.83; N, $24.94 \%$. 5f: Colorless, m.p., $174{ }^{\circ} \mathrm{C}$; from ethanol; yield, $61 \%$; $v_{\max } / \mathrm{cm}^{-1}(\mathrm{KBr}) 3394-3145\left(\mathrm{NH}, \mathrm{NH}_{2}\right) ; \mathrm{m} / \mathrm{z}$ (407); found: C, 70.1; H, 5.3; N, 24.6\%; calcd. for $\mathrm{C}_{24} \mathrm{H}_{21} \mathrm{~N}_{7}$ (407): C, 70.76; H, 5.16; N, 24.08\%. 5g: Colorless, m.p., $127^{\circ} \mathrm{C}$; from ethanol; yield, $73 \% ; v_{\max } / \mathrm{cm}^{-1}(\mathrm{KBr}) 34122-3232\left(\mathrm{NH}, \mathrm{NH}_{2}\right)$; found: $\mathrm{C}$, $68.0 ; \mathrm{H}, 5.1 ; \mathrm{N}, 22.5 \%$; calcd. for $\mathrm{C}_{24} \mathrm{H}_{21} \mathrm{~N}_{7} \mathrm{O}$ (423): C, 68.08; H, 4.96; N, 23.16\%. 5h: Colorless, m.p., $131{ }^{\circ} \mathrm{C}$; from ethanol; yield, $68 \%$; $v_{\max } / \mathrm{cm}^{-1}(\mathrm{KBr}) 3384-3184\left(\mathrm{NH}, \mathrm{NH}_{2}\right) ;{ }^{1} \mathrm{H}$ NMR: 6.57 (s, br, $2 \mathrm{H}$, $\left.\mathrm{NH}_{2}\right) ; 7.00-7.76\left(\mathrm{~m}, 14 \mathrm{H}, \mathrm{C}_{6} \mathrm{H}_{4}, 2 \mathrm{C}_{6} \mathrm{H}_{5}\right) ; 7.33(\mathrm{~s}, 1 \mathrm{H}, \mathrm{CH}) ; 11.22(\mathrm{~s}, 1 \mathrm{H}, \mathrm{NH}) ;{ }^{13} \mathrm{C} \mathrm{NMR:} 113.00$ (C-la); 119.26 (C-3a); $122.30-129.50$ (aromatic-C); $134.00(\mathrm{CH}) ; 137.24$ (C-3); 143.11 (C-4); $\mathrm{m} / \mathrm{z}$ (427); found: $\mathrm{C}, 63.8 ; \mathrm{H}, 4.6 ; \mathrm{N}, 22.6 \%$; calcd. for $\mathrm{C}_{23} \mathrm{H}_{18} \mathrm{~N}_{7} \mathrm{Cl}(427.5): \mathrm{C}, 64.56 ; \mathrm{H}, 4.21 ; \mathrm{N}, 22.93 \%$.

\section{References:}

1. Elgemeie, G. H.; El-Ezbawy, S. E.; Ali, H. A and Mansour, A. K. Bull. Chem. Soc. Jpn., 1994, 67, 738.

2. G. E. H. Elgemeie.; A. H. Elghandour.; A. M. Elzanate., A. M.; and W. A. Masoud. J. Chem. Research (S), 1998, 164.

3. Elgemeie, G. H.; Ali, H. A. and Elzanate, A. M. J. Chem. Research (S), 1996, 340.

4. Elgemeie, G. H; Elghandour, A. H.; Elzanate A. M. and Hussein, A. M. J. Chem. Research (S), 1997, 256.

5. G. E. H. Elgemeie.; A. H. Elghandour.; A. M. Elzanate. and S. A. Ahmed. J. Chem. Research (S), 1998, 162.

\section{Received on March 20, 2002}

